\title{
Pharmacological Treatment for Antipsychotic-Related Constipation
}

\author{
Susanna Every-Palmer ${ }^{*, 1,2}$, Giles Newton-Howes ${ }^{1}$, and Mike J. Clarke ${ }^{3}$ \\ ${ }^{1}$ Wellington School of Medicine, University of Otago, Wellington, New Zealand; ${ }^{2}$ Te Korowai Whāriki, Capital and Coast District \\ Health Board, Porirua, New Zealand; ${ }^{3}$ Centre for Public Health, Queen's University Belfast, Belfast, UK \\ *To whom correspondence should be addressed; Te Korowai Whāriki, Raiha Street, PO Box 50-233, Porirua, New Zealand; tel: +64-918-2733, \\ fax: +64-918-2738, e-mail: susanna.every-palmer@ccdhb.org.nz
}

\section{Background}

Antipsychotic-related constipation is a common and serious adverse effect, especially for people taking clozapine. Clozapine has been shown to impede gastrointestinal motility leading to constipation, and has been reported in up to $60 \%$ of patients receiving clozapine. In rare cases, complications can be fatal. Appropriate laxatives should be prescribed to treat constipation in people taking antipsychotics, but there is a lack of guidance on the comparative effectiveness and harms of different agents in this population. An understanding of the effectiveness and safety of treatment for antipsychotic-related constipation is important for clinicians and patients alike.

\section{Objectives}

To evaluate the effectiveness and safety of pharmacologic treatment (vs placebo or compared against another treatment) for antipsychotic-related constipation (defined as constipated patients of any age, who are treated with antipsychotics, regardless of dose, in which constipation is considered to be an antipsychotic-related side effect).

\section{Search Methods}

We searched the Cochrane Schizophrenia Group's Trials Register (June 15, 2015) which is based on regular searches of CINAHL, BIOSIS, AMED, EMBASE, PubMed, MEDLINE, PsycINFO, and registries of clinical trials, grey literature, and conference proceedings. There are no language, date, document type, or publication status limitations for inclusion of records in this register. We also handsearched bibliographies and contacted relevant authors for additional information.

\section{Selection Criteria}

We included all published and unpublished randomized controlled trials (RCTs) investigating the efficacy of pharmacological treatments in patients with antipsychotic-related constipation. Pharmacological treatment includes laxatives as well as other medicines that could reasonably be used to combat constipation in this population (eg, anticholinergic agents like bethanecol).

\section{Data Collection and Analysis}

Two review authors extracted data from all included studies independently and assessed trials for risk of bias. A third author reviewed $20 \%$ of trials. We analysed dichotomous data using relative risks (RR) and the $95 \%$ confidence intervals $(\mathrm{CI})$. We assessed risk of bias for included studies and used GRADE (Grading of Recommendations Assessment, Development and Evaluation) to create a Summary of findings' table. We discussed any disagreement, documented decisions and attempted to contact authors of studies when necessary.

\section{Main Results}

We identified 2 relevant Chinese studies $(n=480)$ which contributed data to this review. Both studies were over 10 years old and poorly reported, lacking descriptions of contemporary CONSORT reporting prerequisites such as sequence generation, allocation concealment, blinding, participant flow, how the sample size was determined, or how outcomes were measured. The studies also did not report trial registration, pre-specified protocols, consent processes, ethical review, or funding source. We were unsuccessful in making contact with the authors to clarify the missing details. We classified both studies as having an overall high risk of bias.

One study compared glycerol suppository with the traditional Chinese medicine (TCM) approaches of tuina massage and acupuncture. Compared to tuina massage, glycerol laxative was less effective in terms of remaining constipated both 2 days after treament ( $1 \mathrm{RCT}, n=120$, 
$\mathrm{RR}=2.88,95 \% \mathrm{CI}=1.89-4.39$, very low quality of evidence, figure 1$)$ and 3 days ( $1 \mathrm{RCT}, n=120, \mathrm{RR}=4.80$, $95 \%$ CI $=1.96-11.74$, very low quality of evidence). Similar favorable results for acupuncture were observed ( 2 days: $1 \mathrm{RCT}, n=120, \mathrm{RR}=3.50,95 \% \mathrm{CI}=2.18-5.62$, very low quality of evidence), ( 3 days: $1 \mathrm{RCT}, n=120$, $\mathrm{RR}=8.00,95 \% \mathrm{CI}=2.54-25.16$, very low quality of evidence).

The other study compared mannitol, an osmotic laxative with rhubarb soda or phenolphthalein. Mannitol was more effective than rhubarb soda or phenolphthalein in terms of the risk of remaining constipated within
24 hours of treatment ( $1 \mathrm{RCT}, n=240, \mathrm{RR}=0.07,95 \%$ $\mathrm{CI}=0.02-0.27$, very low quality of evidence).

No data were reported for our other important outcomes: need for rescue medication, bowel obstruction (a complication of antipsychotic-related constipation), quality of life, adverse events, leaving the study early and economic costs.

\section{Conclusions}

We had hoped to find clinically useful evidence appraising the relative merits of the interventions routinely used

\subsection{Glycerol versus tuina}

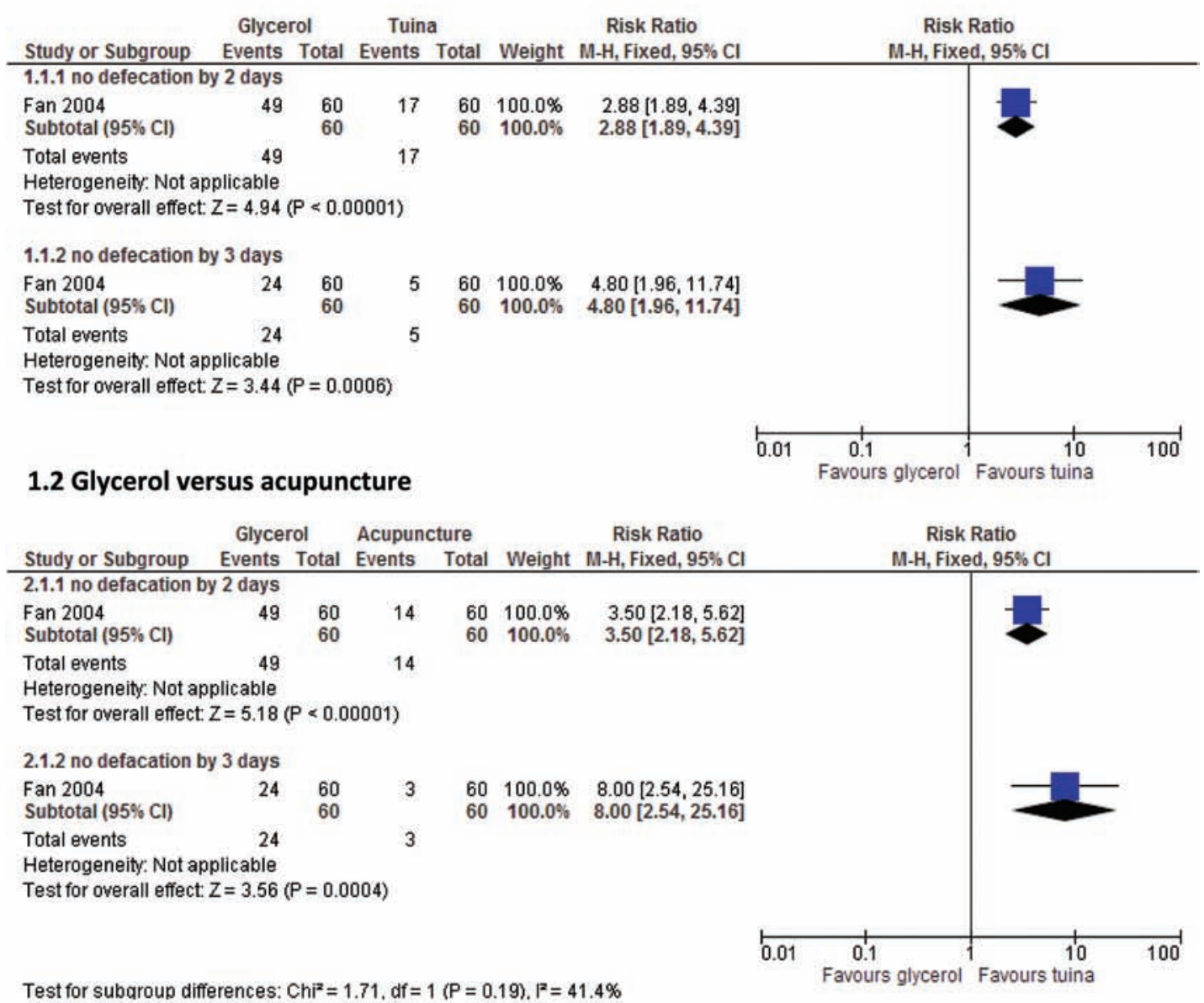

1.3 Mannitol versus phenolphthalein or rhubarb soda

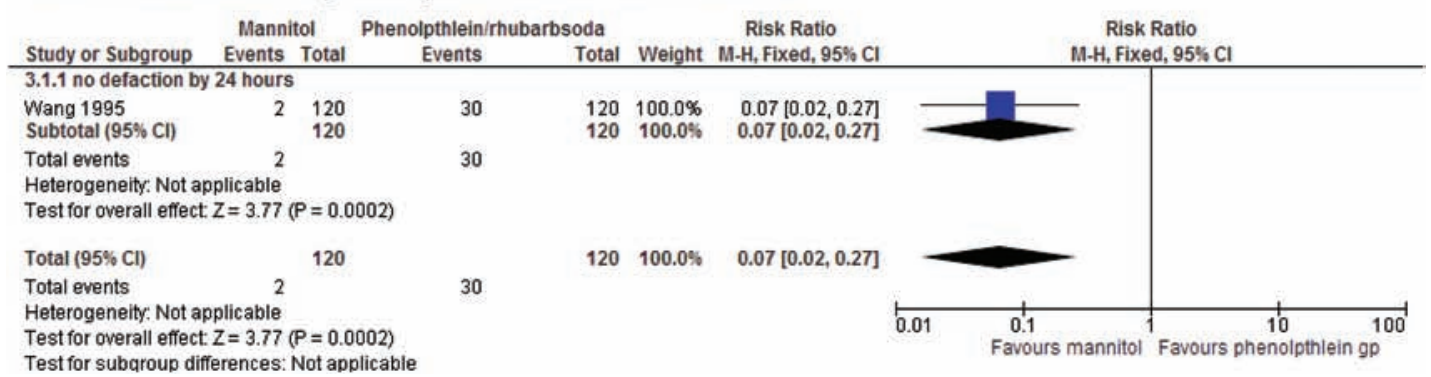

Fig. 1. Global or clinical change in constipation. 


\section{S. Every-Palmer et al}

to manage antipsychotic-related constipation, a common and potentially serious adverse effect of the use of these drugs. The results were disappointing. There were no data comparing the common pharmacological interventions for constipation, such as lactulose, polyethylene glycol, stool softeners, lubricant laxatives or of novel treatments such as linaclotide. Data available were very poor quality and the trials had a high risk of bias. Data from these biased studies suggested that in terms of "still constipated," mannitol, an osmotic laxative, was more effective than rhubarb soda and phenolphthalein, and a 2-week course of glycerol suppositories was less effective than the TCM approaches of tuina massage and acupuncture.

Overall, there is insufficient trial-based evidence to assess the effectiveness and safety of pharmacological interventions for treating antipsychotic-related constipation, due to limited poor quality data (few studies with high risk of bias and no meta-analyses). The methodological limitations in the included studies are obvious, and any conclusions based on their results should be made with caution. Methodologically rigorous RCTs evaluating interventions for treating antipsychotic-related constipation are needed. For details please see full Cochrane review. ${ }^{1}$

\section{Reference}

1. Every-Palmer S, Newton-Howes G, Clarke MJ. Pharmacological treatment for antipsychotic-related constipation. Cochrane Database Syst Rev. 2017; 1:CD011128. 